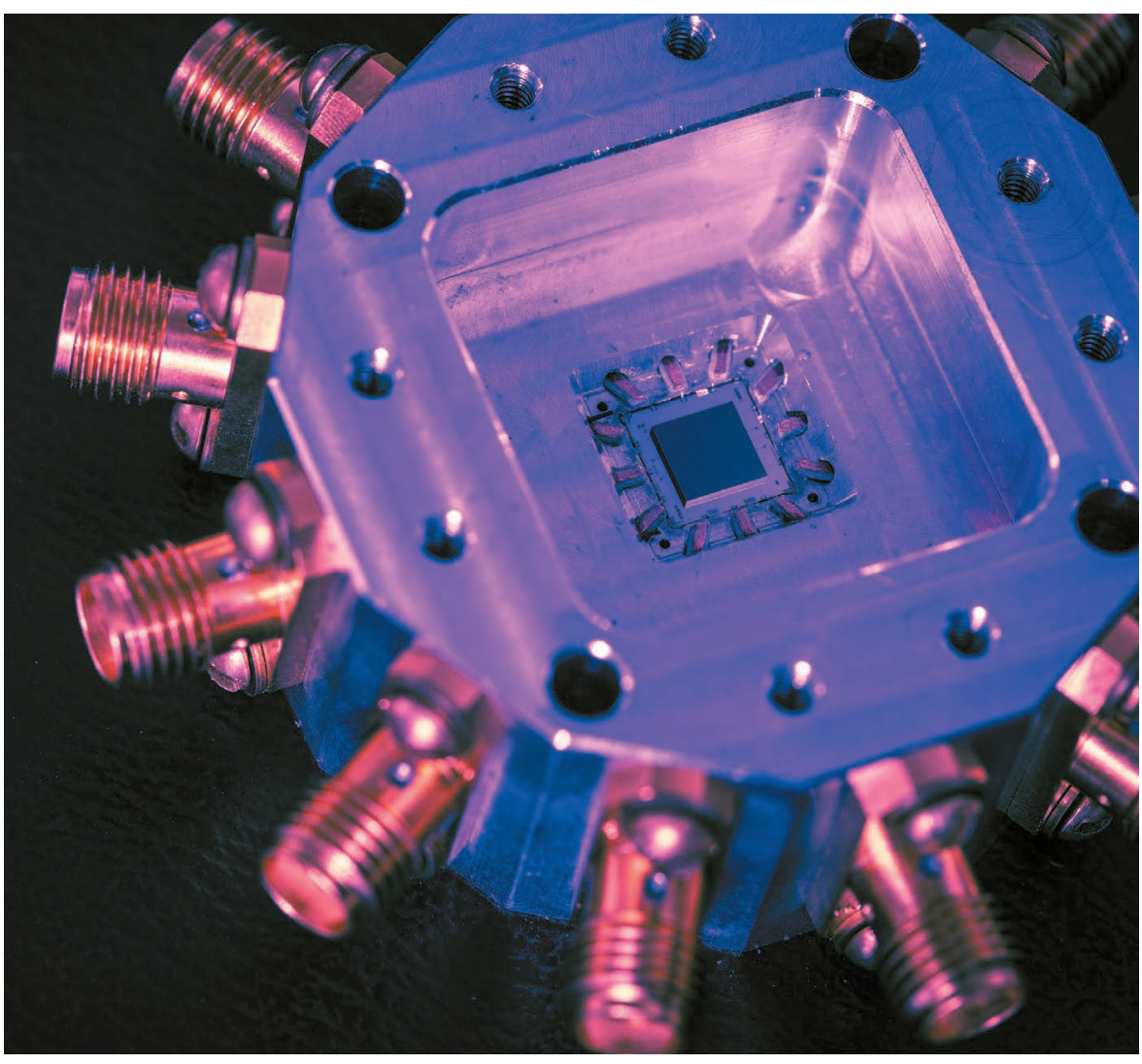

A computer chip created by Google Research to manipulate data using quantum bits (qubits).

QUANTUM PHYSICS

\section{A trek through the probable universe}

I takes care to handle the paradox of describing something that no one truly understands. The fastidious, plainspoken science writer and former Nature editor Philip Ball mostly achieves this ambition in his 23rd book, Beyond Weird, a clear and deeply researched account of what's known about the quantum laws of nature, and how to think about what they might really mean.

Quantum theory, developed in the early twentieth century by Max Planck, Albert Einstein, Niels Bohr and other luminaries, strips 'reality' of its usual meaning by seeming to say that nothing is certain about atoms or elementary particles until they are measured. A particle's location, for example, is a matter of probability. It is defined by a spread-out, abstract 'wavefunction' until the act of looking for the particle somehow collapses the wavefunction to one spot, inducing a single reality. We inhabit, as theoretical physicist John Wheeler said, a "participatory universe" - and confusion stems from there. Ball tells us that quantum theory is "cognitively impenetrable". Yet he convincingly argues that the questions about it have sharpened and even changed profoundly over time - progress, in lieu of answers.

Along with the historic discoveries, Ball brings readers up to speed on today's "quantum renaissance". This active intellectual period is fuelled by quantum-computing research and the rise of quantum information theory, pioneered by researchers including David Deutsch, Peter Shor and Charles Bennett. Quantum mechanics is now seen as, more than anything, a set

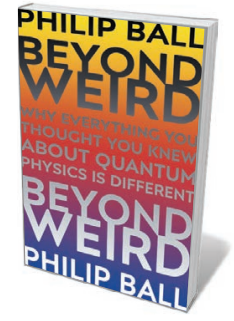
Why Everything You Thought

You Knew About Quantum Physics

Is Different

PHILIP BALL
Beyond Weird:

Bodley Head: 2018

of rules about how information can be shared and processed. That, Ball says, is why quantum computing has proved so stimulating: what's possible and impossible to compute "follow from the same rules that govern what is knowable and what is not". Additionally, physicists, starting with Dieter Zeh in the 1970s and Wojciech Zurek in the 1980s, have developed a decent understanding of the quantum-classical divide: the reason particles can exist in superpositions of many possible states, but cats are only ever dead or alive. Classical physics is now seen as emerging from underlying quantum laws because of 'decoherence' That is, brushes with the environment cause quantum superpositions to lose coherence as information about them leaks out - and the bigger the system, the faster it happens.

Modern theoretical studies and exquisitely controlled experiments have revealed the newly important role of principles such as information causality, which puts a hard limit on the amount of information that can be deduced about a quantum system after it has been measured. Researchers in quantum reconstruction are taking the new insights and going back to basics, looking for the minimal set of axioms from which the full range of quantum behaviours emerges

Ball is an intelligent guide. The weirdness is dizzying at times, however, and landmark discoveries might be missed on his meandering tour of non-intuitive phenomena and their implications. He gives a rather low-impact description of the double-slit experiment. Yet this is arguably the most instructive demonstration of quantum weirdness: a particle behaves like a wave that passes through both slits in a screen and interferes with itself on the other side, producing peaks of probability in the places where it might then be found. Also lacking oomph is the description of Bell's theorem, the 1964 watershed that enabled physicists to prove experimentally that entangled quantum particles exhibit 'non-locality'. That finding upended the classical belief that things must exist in places.

Other passages are riveting, such as Ball's elaboration of the fascinating debate surrounding the meaning of the wavefunction. As he writes, efforts to crack quantum mechanics have forced scientists to examine ancient philosophical questions about the nature of reality, knowledge and existence. Do quantum wavefunctions exist, for instance, or do these probabilistic descriptions of 
the world reflect our own ignorance?

Ball cites the Copenhagen interpretation, the hard line taken by deep-thinking Danish physicist Niels Bohr, who said, "There is no quantum world. There is only an abstract quantum physical description." Bohr also noted that physics concerns not how nature is, but "what we can say about nature". (Theoretical computer scientist Scott Aaronson has defined Copenhagenism as "shut up and calculate except without ever shutting up about it”.) Ball sees Bohr's perspective as frustratingly elusive.

The competitors hardly fare better. The de Broglie-Bohm interpretation, which imagines particles guided by invisible pilot waves, is, in Einstein's words, "too cheap". Stochastic-collapse models equip wavefunctions with extra components that randomly choose their states at the moment of measurement - "a bodge", according to Ball. The consistent-histories interpretation, which says that anything that happens has to be consist-

"Ball is at his

liveliest when

incisively

critiquing the

many-worlds

interpretation." ent with what came before, he sees as useful, although he also says that it fails to supply "a physical picture". A theory known as QBism takes an even harder line than Copenhagenism, interpreting quantum wavefunctions as personal beliefs - but it does claim compellingly that reality, rather than being rigid and deterministic, exhibits creativity or novelty, "almost a lawlessness out of which laws can arrive".

Ball is at his liveliest when incisively critiquing the many-worlds interpretation. This popular hypothesis holds that every measurement sends the Universe along branching paths, proliferating the number of parallel realities. Logical problems arise, he argues, when you assume that every quantum probability plays out - including probability itself becoming meaningless.

No angle on the quantum world feels satisfactory, but many add insight. There's a sense of growing perspective in a haze of confusion. Beyond Weird distinguishes itself by giving life to the ideas, rather than just to Einstein, Bohr, Erwin Schrödinger and the other colourful quantum pioneers. Their discoveries and quandaries about spooky action at a distance and feline superpositions were so deliciously disorienting that they've been endlessly rehashed by other authors. Ball brings them all into the prose, but in service of the science. "Quantum theory had the strangest genesis," he says. "Its pioneers made it up as they went along. What else could they do?"

Natalie Wolchover is a physics reporter and senior writer for Quanta Magazine. She lives in Brooklyn, New York.

e-mail:nwolchover@quantamagazine.org

\section{Books in brief}

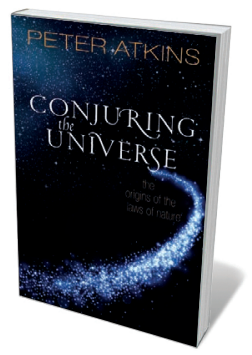

Conjuring the Universe: The Origins of the Laws of Nature Peter Atkins OXFORD UNIVERSITY PRESS (2018)

It's rare to find a study of physical laws that is also a bravura display of rarefied humour and experiential depth; but such is this gem by chemist Peter Atkins. Looking at both 'inlaws' and 'outlaws' - the deep structural laws of the cosmos and minor elaborations such as Robert Hooke's law of elasticity - Atkins explores the conservation of energy; laws related to temperature, electricity and magnetism; and fundamental constants such as the speed of light. He also shows, in one mind-bending chapter, how something really did come from nothing at the moment of cosmogenesis.

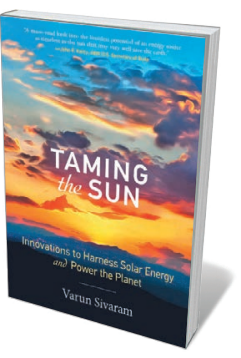

Taming the Sun

Varun Sivaram MIT PRESS (2018)

In 2017, solar power became the fastest-growing new-energy source. Yet as energy-policy specialist Varun Sivaram details in his global survey, that surge will be unsustainable unless countries invest in solar innovation on three fronts: financial (to amass investment); technological (to capture and store solar energy); and systemic (to re-engineer electricity grids). Solar power will also have to grow 30-fold by 2050. Sivaram includes a raft of case studies, from current research on the photovoltaic materials called perovskites to Off Grid Electric, a start-up aiming to electrify swathes of Africa by 2019.

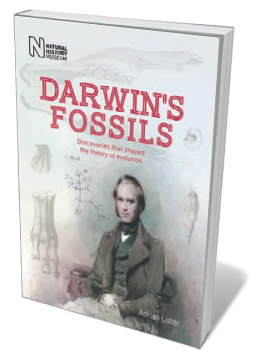

\section{Darwin's Fossils}

Adrian Lister NATURAL HISTORY MUSEUM (2018)

A "young man of promising ability, extremely fond of geology". That accolade given to Charles Darwin by the captain of the HMS Beagle, Robert FitzRoy, propelled the fledgling scientist on the ride of his life. As naturalist on the ship's 1831-36 voyage to South America, Darwin came into his own as a geologist, illuminating the formation of coral reefs and continental uplift. And, as palaeobiologist Adrian Lister details in this deft, beautifully illustrated account, no less important were Darwin's discoveries of gargantuan mammal fossils — including the 1.5-tonne giant sloth Mylodon darwinii.

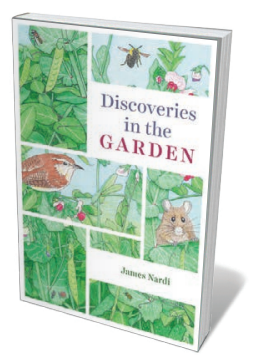

Discoveries in the Garden

James Nardi UNIVERSITY OF CHICAGO PRESS (2018)

Biologist James Nardi urges us to recall that it's a jungle out there - in the back garden, that is. His companion guide to garden science is a learned romp through plant biology; solar energy and soil nutrients; the movement of vines; and "fellow gardeners", from single-celled protozoans to beetles. Here, too, are experiments on the basics, such as photosynthesis, and a wealth of stunning images. These range from a prickly study of weed burrs to a scanningelectron-microscope shot of trichomes, hairs that stud tomato leaves like "dark lollipops" and give the plant its pungent odour.

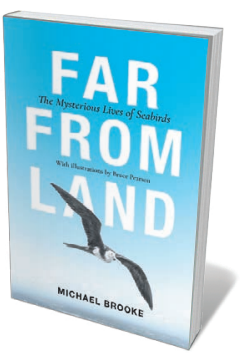

Far from Land: The Mysterious Lives of Seabirds

Michael Brooke PRINCETON UNIVERSITY PRESS (2018)

For 40 years, ornithologist Michael Brooke has traversed the seas in pursuit of seabirds - from Alexander Selkirk Island west of Chile to the Shiant Isles of the UK Hebrides. His tour of the ecological highlights is gripping in scope and in granular detail gleaned from observations of puffins, penguins, fulmars and more. Brooke begins at fledging and glides on through migration, navigation, breeding and myriad threats facing these avian wonders, from introduced predators and light pollution to overfishing and ocean plastic. Barbara Kiser 